\title{
La letteratura versus la televisione: il caso di Nove e Covacich
}

Hanna Serkowska

\section{(2) OpenEdition}

Journals

Edizione digitale

URL: http://journals.openedition.org/cei/140

DOI: $10.4000 /$ cei. 140

ISSN: 2260-779X

Editore

UGA Éditions/Université Grenoble Alpes

\section{Edizione cartacea}

Data di pubblicazione: 15 juin 2010

Paginazione: 215-223

ISBN: 978-2-84310-168-7

ISSN: 1770-9571

Notizia bibliografica digitale

Hanna Serkowska, «La letteratura versus la televisione: il caso di Nove e Covacich», Cahiers d'études italiennes [Online], 11 | 2010, online dal 15 décembre 2011, consultato il 27 mars 2021. URL: http:// journals.openedition.org/cei/140 ; DOI: https://doi.org/10.4000/cei.140 


\title{
LA LETTERATURA VERSUS LA TELEVISIONE: IL CASO DI NOVE E COVACICH
}

\author{
Hanna Serkowska \\ Université de Varsovie
}

«[...] è ancora possibile fare esperienza diretta delle cose in un mondo di simulazioni e simulacri?»

Filippo La Porta, La nuova narrativa italiana, 2003, p. 260.

Il primo Calvino, quello de $I l$ sentiero dei nidi di ragno, dimostra quella vitalità e quell'interesse verso il «mondo non scritto» che presto si andrà sfibrando, esaurendo, complicando. Il Calvino degli anni quaranta che si lamenta che la letteratura manchi di freschezza e di vita, che non vada oltre l'esperienza cartacea, sarà presto rimpiazzato dal suo contrario attento precipuamente al «mondo scritto». Il signor Palomar non esprime in modo diretto le sue posizioni nei confronti del mezzo (tivù) che gli intellettuali come Pasolini rendevano colpevole del "genocidio culturale», e l'oggetto del presente saggio è la reazione di alcuni giovani scrittori negli anni '9o al danno che si sarebbe già consumato. Ritengo tuttavia utile iniziare evocando il concetto del realismo che ha lo scrittore sanremese e su cui si accentrano alcune incomprensioni, o semplificazioni nel dibattito tuttora in corso attorno alla cosiddetta "letteratura dell'inesperienza». Antonio Scurati che nel suo recente saggio eponimo muove da una constatazione, a parer suo funesta, che la letteratura avrebbe perduto la relazione con l'esperienza, intesa come esperienza del mondo reale. Parafrasando Manganelli che difendeva la letteratura dalle accuse di immoralità, mi domando: se la letteratura ha mai avuto relazione con l'esperienza del mondo reale? $\mathrm{O}$ forse il realismo nella letteratura è un'altra cosa? 
Se è vero che - come diceva Baudrillard - la televisione col suo esasperato (cercato) realismo, o con l'iperrealismo di fatto, perde il reale nel momento stesso in cui lo ritrova, forse è corretto dire che la letteratura incontra il reale mentre lo perde, nel senso di liberarsi della volontà di rimanere a contatto diretto col reale. Diventa pertinente la rivalutazione che del rapporto fra letteratura e esperienza ideava il secondo Calvino, quello che, come dice Carla Benedetti, ebbe fede «in un'idea restrittiva della letteratura» (Benedetti, p. 2I). Sarebbe sbagliato leggere la distinzione calviniana in letteratura e mondo d'accordo con le poetiche realistiche (che attribuiscono alla letteratura il compito di rappresentare il mondo). La letteratura è per Calvino, barthesianamente, «intransitiva», ovvero si riferisce soprattutto a se stessa. Per una tale depotenziata letteratura il reale è solo un pretesto, ed essa si trova sempre con il mondo in un «rappporto a distanza», non può spiegare il mondo (Benedetti, p. II6). La scrittura letteraria incontra quindi il mondo come problema: il mondo è ciò che essa non può non supporre per continuare a funzionare (Benedetti, p. II8). Anche la scrittura come gioco non può fare a meno di pensarsi in relazione ad un mondo ad essa esterno, e proprio quando sta per pensarsi incapace di agire nel e sul reale, e inutile, superflua, si rivela capace di testimoniare, spiegare, insegnare. E nel far sì «riporta [in modo diverso, ma nell'unico modo possibile] la letteratura al mondo» (Benedetti, p. 132). Nessuna meraviglia che alcuni seguaci del Calvino di Palomar (Paola Caprilo, Andrea De Carlo, Daniele Del Giudice, Mario Fortunato, Dario Voltolini) avrebbero cercato di eludere il richiamo della Gorgonie di cui temevano lo sguardo pietrificante avvicinandola indirettamente, cercando mediazioni, trasformando il reale, cifrandolo per mezzo di metafore, visioni oniriche, allontanandosene.

Tenendo a mente quanto precede, l'ottica di Antonio Scurati (quella del timore della "fine di ogni realismo»), per cui il mondo oggi è per noi ormai soltanto «l'assenza di un mondo» (non abbiamo l'esperienza del mondo che viviamo) e di conseguenza non sappiamo tradurre quel mondo in un mondo di parole, simboli e immagini quale è la letteratura, suscita alcune riserve. Per quanto si può essere d'accordo con la prima ipotesi (in seguito cerco di spiegarla in modo più articolato), riformulata nei termini di Baudrillard, la seconda supposizione resta infondata, cosa che s'intende dimostrare richiamandosi ai testi di alcuni autori - quali Aldo Nove e Mauro Covacich - di quella che chiamerei letteratura dell'esperienza.

Scurati arguisce che se viene alterato e reso assente il mondo, annullate le distanze fisiche, stravolta la percezione tradizionale, abolita la distinzione tra vicino e lontano, cancellata la temporalità vissuta che è la fon- 
damentale dimensione antropologica, la colpa ne è delle tecnologie della telecomunicazione e della telepresenza. Insomma, «l'inesperienza è la nuova forma di indigenza, il nuovo senso di nullatenenza assoluta» (Scurati, p. 34). Si può essere d'accordo con Scurati quando afferma che l'inesperienza cresce in proporzione alla diffusione dei mezzi di comunicazione di massa, soprattutto con il trionfo della neotelevisione che mentre ci dà un'immagine del reale prefabbricata allo stesso tempo nega il proprio statuto di rappresentazione. Pare invece difficilmente condivisibile l'ipotesi che gli scrittori non riescano a tenere fede al richiamo del reale, a rappresentarlo tale e quale.

Diciamo per inciso che Scurati sorvola quei rischi legati all'invasione del campo della letteratura da parte della televisione che ravvisa tra gli altri Giulio Ferroni. Lo studioso non suona allarme quando la letteratura è in genere invasa da codici altri, considerato che il terreno della letteratura ha sempre subito molteplici interferenze, si è lasciato attraversare e contaminare. Quando tuttavia l'invasione è operata dalla televisione, c'è di che preoccuparsi perché la televisione «annulla completamente la dimensione che è essenziale per la stessa sopravvivenza della letteratura» (Ferroni, p. 93). A Ferroni sta a cuore l'azione perniciosa che la televisione compie promuovendo la cattiva letteratura, piegando la letteratura alle proprie aspettative, imponendo una scala di valori e una serie di modelli che emanano dallo schermo. In base a quei valori e modelli, poi, gli editori pubblicano e sostengono libri di personaggi televisivi (comici, attori, cantanti, politici, giornalisti), «libri che spesso arrivano in testa alle classifiche, e nei giovani lettori si riafferma l'idea che la letteratura possa essere interessante solo se concepita come una sorta di appendice o di supporto ad altre forme di intrattenimento» (Ferroni, p. 95). Alla nostra attenzione si impongono soltanto quegli scrittori che sanno offrire, attraverso la tv, la propria immagine e la propria presenza, valgono solo quelli di loro che sanno passare in televisione. La preoccupazione di Scurati sembra riguardare invece più generalmente quei contenuti della letteratura di oggi che - dietro l'impulso della televisione - smarriscono il rapporto con il reale, e come la televisione, imitandola, ci allontanano dal mondo dell'esperienza.

Lo scrittore di Correggio, dai primi anni ha predicato a sé e agli altri (penso tra l'altro al laboratorio Under 25) la fede della scrittura all'esperienza. La scrittura doveva nascere (era stilizzata?) dall'esperienza del reale. Scrivete come vivete, quello che vivete, quello che siete, come siete, consigliava ai giovani adepti della scrittura. Così Tondelli è riuscito a comunicare sé agli altri, a dar conto di sé in quanto individuo inserito nel contesto nel quale viveva, il proprio esistere in questo mondo con tutte le inquietudini, 
ansie e angosce che il suo tempo gli comunicava. Rappresentando se stesso, egli sosteneva, uno scrittore può meglio rappresentare l'umanità del suo tempo. Tondelli è stato imitato in questo da tutta una schiera di vari Ammaniti, Brizzi, Caliceti, Nove, Scarpa e altri, definiti di volta in volta pulp, cannibali, narrative invaders, terza ondata, neo-neoavanguardisti, scrittori dell'eccesso o dell'orrore estremo, presto stroncati e liquidati come una generazione di scrittori che ignorano la precedente letteratura italiana, scrivono dopo la morte della letteratura, fanno leva sulle pulsioni primordiali incanalate in preordinati stereotipi di massa. Eppure mi pare che vi sia dell'altro nelle scritture di quei giovani scrittori degli anni ' 90 che parlano dell'universo giovanile, costituito da televisione, merci, consumi, supermercati, stazioni ferroviarie, treni, bar, discoteche, scuole, caserme, località balneari, musiche, marchi, loghi, videogiochi, fumetti, radio, film, e ancora una volta la televisione. Vi si raccontano le condizioni di vita dei giovani, i conflitti generazionali, stili di vita, emozioni, sentimenti e non da ultimo la disgregazione della famiglia di cui è colpevole la tivù che costituisce il mostruoso centro della vita domestica, ipnotizza $\mathrm{e}$ distrae. Tondelli imputava infatti i massmedia non solo della crisi affettivofamiliare, ma anche di trasmettere un'immagine deformata dell' universo e con ciò di rafforzare l'idea di una generazione allo sbando, stretta fra mitologie consumistiche e un vuoto emozionale. Per contrastarla ha deciso di prendere parola «dall'interno del mondo giovanile», e di passare agli altri giovani l'invito a descrivere le loro esperienze (Mondello, p. 9).

Perché invece non raccontate quello che fate, che sentite: i vostri tormenti, i vostri rapporti a scuola, con le ragazze, con la famiglia. [...] C'è bisogno di sapere tutte queste cose. Siete gli unici a poterlo fare. Nessun giornalista, per quanto abile, potrà raccontarle al vostro posto. Nessuno scrittore. (Tondelli, pp. 685-686.)

Di conseguenza quanto loro (e il loro caposcuola) hanno scritto è talmente immerso nel mondo reale che leggendo se ne ricava, meglio che da un trattato di sociologia, un quadro circostanziato degli anni in cui sono ambientate le loro trame. Non a caso alla domanda perché si scrive? Tondelli usava rispondere di volta in volta: per assimilare la realtà, per filtrarla mettendola in scena. La scrittura è un mangiare la realtà e digerirla nel senso di nutrirsene, per poterla anche, scrivendo, sopportare, sostenere.

Il gesto dei seguaci di Tondelli a me pare privo di quel cinismo e vuotaggine che gli si contestava, in quanto secondo alcuni invece avrebbero abdicato alle loro prerogative di scrittori e di intellettuali, di non assumere un punto di vista, non giudicare. Sostengo al contrario che il loro è un intervento non acritico sul mondo reale di cui si sentono angustiati. Un esempio vistoso del rifiuto della cultura monopolizzata dalla neote- 
levisione sono i racconti di Aldo Nove Woobinda e Superwoobinda che in ben 40 puntate rispecchiano a perfezione la sua subdola azione, e nel rispecchiarla, la ripudiano. Il centro di quell'universo è costituito dalla televisione, la sindrome televisiva, e i danni che essa arreca agli spettatori. La tv diventa il bersaglio di Nove che ne contesta l'azione anestetizzante (nel senso di insensibilizzazione, di oscenità) basata sull'eccesso e sulla saturazione. Per avversare l'assuefazione lo scrittore ha abolito i legami di causa ed effetto, preferendo la casualità alla causalità. Non solo le tematiche, ma anche la strategia narrativa imita la tv, in particolare lo zapping, la ripetizione, il riuso, e il riassemblaggio dei materiali già utilizzati, materiali di riporto.

Conviene precisare che Nove accusa il mezzo ubiquo e onnivoro di due torti. Il primo è il cattivo esempio, il saturarci con notizie sconvolgenti, espressioni scurrili, immagini cruente, violente o oscene. Qui la vena moralistica di Nove trae certamente da Karl Popper che postulava una patente per fare la tv considerata "cattiva maestra» in quanto portatrice della violenza in case ove altrimenti violenza non ci sarebbe, mentre essa dovrebbe compiere un ruolo di educazione (quel ruolo che essa si arroga comunque, anche quando educa male). Il secondo rischio che la televisione ci fa correre chiama in causa il teorico francese Jean Baudrillard, e consiste nell'azzeramento dell'universo non (tele)mediato, che la tivù priva di realicità. Aldo Nove fa vedere come funziona questo meccanismo. In Puerto Plata Market afferma che anche i sentimenti quali amore per essere esperiti e compresi necessitano la mediatizzazione: è «meglio se senza nemmeno parlare tu ti prendi in casa chiunque e insieme guardate la stessa cosa in televisione pensando che è vera. Quel momento è l'amore perfetto", perché "l'amore perfetto è quando tutto va come un film» (Nove, 1997, p. 7). Apparentemente si scredita l'empirìa a favore di un rapporto indiretto, mediato con le cose. La presunta entusiastica predilezione per uno sguardo mediato costituisce, in verità, la denuncia della sornione derealizzazione dei simulacra teletrasmessi e il loro sostituirsi all'universo reale. Nel racconto intitolato Ruanda Nove scrive:

Considerato che ora ho un televisore ventiquattro pollici subacqueo posso vedere il Ruanda in fondo alla piscina [...] Posso vedere il Ruanda quando vado in Milano con la mia Cherokee [...] posso vedere ogni genere di morti mentre parcheggio. Quando vado in montagna con il mio televisore da polso ogni tanto mi fermo ad ammirare il paesaggio, mangio qualcosa e guardo il Ruanda. (Nove, I998, p. 58.)

La violenza dei media di cui parla Baudrillard non si riferisce - come in caso di Popper - al contenuto delle immagini (ed è in questa chiave che 
leggo Aldo Nove). La violenza dell'immagine uccide il reale facendolo sparire dietro di sé. La tv nei testi di Nove non riproduce e non rappresenta il reale, bensì lo produce o lo rimpiazza. Le immagini che essa ci rimanda sono bloccate nella loro intransigenza, nell'autoreferenzialità. Non rappresentano, bensì mascherano e simulano l'inesistenza del reale e cercano di sostituirsi ad esso. La tv all'interno della prosa noviana compie, infatti, degli atti definiti da Baudrillard atti semiurgici, essa produce segni senza referenza, significanti senza significato. Il reale, manipolato ed eclissato, è ridotto ai meri simulacra, alle immagini con cui si confonde, ed è questo che la prosa di Aldo Nove ci invita a riconoscere. Nel racconto sulla strage in via Palestro il protagonista è completamente sbalordito quando constata l'esistenza, anzi la preesistenza di una referenza alle immagini rimandate dalla tv. Constata allora che il reale è più sbiadito dei suoi simulacra, meno credibile, forse inesistente, manipolato, inventato, e che egli vi preferisce le (più affidabili) immagini rimandate dalla televisione: "Quando sono andato alla strage di via Palestro [...] passando tra la gente, mi vedevo le macerie ed ero triste, ma meno che guardando la televisione, perché alla televisione tutto è sempre più vero.» (Nove, I998, p. 46.)

Possiamo concludere che la tv non rimanda il reale ma solo le immagini che lo eclissano, che creano nell'insieme un mondo ipersemplificato, preselezionato, livellato e ri-gerarchizzato. Lo si vede con chiarezza nel romanzo di un altro giovane scrittore, Mauro Covacich, intitolato Fiona. Il protagonista del romanzo, Sandro è al contempo autore di un popolare reality show televisivo (intitolato Habitat, un equivalente del Grande Fratello) e un bombarolo che prepara ordigni esplosivi e li immette nei supermercati non per uccidere delle persone che infatti ne escono solo graffiate o leggermente ferite, quanto invece per ingaggiare una scommessa con l'impero del visibilio che il «volto diurno» del protagonista contribuisce a creare. Mentre entra nel supermercato Sandro gioca a non lasciarsi riprendere dalle telecamere commerciali il cui occhio panottico (constatato che non vi sono più nel mondo di oggi oasi non videomonitorate) ci spia dalla mattina alla sera e ci attira mentre rimanda la nostra immagine su qualche monitor circostante, al punto di far preponderare le immagini digitali alla nostra vera presenza e identità. E così, per esempio, alla domanda come sono padre e figlia, protagonisti del romanzo, il narratore di Covacich ribatte che essi sono ripresi e trasmessi, offerti in chiaro, sgranati, saturi, un po' schiacciati (Covacich, p. 5). Col passar del tempo, dunque l'occhio a cui nulla sfugge diventa un nemico di Sandro che cerca di compiere atti che potrebbero passare da esso inosservati, e con l'occasione, mentre provoca l'esplosione, spacca la telecamera e nebulizza la 
visione. Finisce per sabotare il sistema di cui egli è in parte autore. Quel che fa è dunque una sfida all'impero dell'immagine.

La tivù diventa il bersaglio di Sandro non solo perché invade la privacy delle persone, perché veicola contenuti volgari (la tivù spazzatura), ma innanzi tutto perché mentre finge di trasmettere eventi, fa accadere cose secondo il suo preciso disegno. Sdoppia il reale, lo falsa, contraffa. Mentre i concorrenti di Habitat si industriano, infatti, a vivere in diretta, sullo schermo e davanti agli occhi degli spettatori, gli autori del programma si segnano note e riferimenti "per i ventiquattro minuti di montato della puntata in chiaro di domani e passandosi continuamente il regista al telefono" (Covacich, p. 13), ogni tanto convocano i concorrenti nello stanzino detto confessionale, e gli passano in continuazione istruzioni, suggerimenti, ordini finalizzati ad incrementare e a tenere alti i dati di audience. E quindi mentre Covacich ci fa capire come il tutto sia pilotato, orchestrato, montato nello studio, indica che proprio il programma in cui la tivù fa credere di togliersi di mezzo, in cui finge di rimuovere ogni filtro di mediazioni, e di giocare "al reale" (si tratta per l'appunto di un reality show) mente, falsa, contraffa. Niente è autentico, vissuto, tutto costruito secondo un preciso scenario. Per richiamare la nostra attenzione su questo fatto, Covacich poi opera un apposito straniamento facendoci notare come i suoi protagonisti (tutti gli spettatori?) hanno perso contatto con il mondo reale, naturale, e come per capire quel mondo si ha bisogno di una mediazione da parte del mondo innaturale. Vediamone un esempio: le bacche dei lecci tra cui si stende l'amaca scoppiettano, ma dire ciò non basta, chi racconta si rende conto che pochi oggi sanno che rumore emettano le bacche dei lecci calpestate. Per spiegarlo deve ricorrere a una similitudine: esse scoppiettano come imbottitura da impacco sotto i pneumatici dell'Audi. Per farci esperire qualcosa del tutto naturale e immediato si ha bisogno di un elemento innaturale (la plastica a bolle da imballaggio) che ci è più nota delle bacche dei lecci. Similmente un termine di paragone diventa varie volte una scena di un film o di un programma televisivo, altrimenti non riusciremmo a spiegarci più nulla.

Nel romanzo di Covacich, insomma, la televisione, e più latamente il mondo dell'immagine diventa responsabile della nostra odierna perdita dell'esperienza del reale mondo naturale delle cose, e perciò stesso essa è scelta come bersaglio, meccanismo da smontare, nemico da disarmare.

A questo punto però il cerchio si chiude, quella di Nove, Covacich, Montesano, Culicchia, Caliceti, Balletra, Santacroce e molti altri può essere considerata letteratura dell'inesperienza nella misura in cui il mezzo che derealizza il reale costituisce l'oggetto, il tema, e anche il modo (penso alla 
strategia narrativa che imita lo zapping) del narrare, dello scrivere. D'altro canto, proprio perché la tv è il centro che domina la vita e il mondo odierni che diventano l'oggetto della rappresentazione, dobbiamo definire questa una letteratura dell'inesperienza, come fa Scurati?

Richiamiamo brevemente anche la soluzione che contro i mezzi, e in particolare contro il mezzo, propone in $A$ passo di gambero l'ex-paladino e difensore della comunicazione (e della cultura) di massa Umberto Eco anche se con ogni probabilità il motivo che lo ha spinto a farlo era il suo scetticismo verso quei meccanismi che riguardano i mezzi controllati (che sono poi quasi tutti in Italia) da Silvio Berlusconi. I mass media, avverte il semiologo, danno a chi ne dispone il potere illimitato. Basta escludere dalle trasmissioni televisive il proprio avversario per sconfiggerlo, condannarlo alla non esistenza. L'opinione pubblica se ne scorda presto, ed è come se fosse morto. Similmente, basta lamentarsi ad alta voce e naturalmente tramite la televisione, di essere criticati dai mass media affinché la gente sia convinta che vi siano ancora mezzi avversi al governo, perché tale è l'effetto di realicità prodotto dalla notizia teletrasmessa. Infine propone Eco un brave new game, un gioco chiamato come l'inno nazionale Fratelli d'Italia, un gioco più perfetto del Grande Fratello perché potenzia al massimo il senso del voyerismo e del pettegolezzo. Le telecamere, ubicate ovunque in ogni spazio pubblico e privato, riprenderebbero tutti i cittadini di modo che possiamo, attimo dopo attimo, seguire la vita di sessanta milioni di persone. Se però ciascuno desidererà mettersi in mostra e per superare gli altri si sforzerà di agire meglio degli altri, vi sarà ancora chi avrà tempo di guardare la tv? Soltanto così, sentenzia divertito Eco, si potrà disarmare il pericoloso mezzo con i suoi stessi metodi.

Concludendo va detto che alcuni giovani scrittori degli anni '9o in Italia hanno scelto una letteratura macro- o meta-realista nel senso che hanno ritratto il reale di cui è parte anche l'indebolimento dell'esperienza umana delle cose e il pericolo che la tivù ci fa correre quotidianamente di usurparsi il posto del demiurgo e di produrre lei prodotti esistenziali manipolando le nostre vite. Tuttavia, proprio finché ci sono scrittori come Nove e Covacich, la tivù ha un serio rivale, un nemico che, la denuncia, la smonta, la decostruisce e Scurati non si deve preoccupare. 


\section{Bibiografia}

Barthes Roland, «Scrittori e scriventi», in Saggi critici, Torino, Einaudi, 2002.

Benedetti Carla, Pasolini contro Calvino. Per una lettura impura, Milano, Bollati Boringhieri, 1998.

Covacich Mauro, Fiona, Milano, Einaudi, 2005.

Eco Umberto, A passo di gambero. Guerre calde e populismo mediatico, Milano, Bompiani, 2006.

Ferroni Giulio, I confini della critica, Napoli, Alfredo Guida Editore, 2005.

La Porta Filippo, La nuova narrativa italiana, Torino, Bollati Boringhieri, 2003.

Mondello Elisabetta, In principio fu Tondelli. Letteratura, merci, televisione nella narrativa degli anni novanta, Milano, Il Saggiatore, 2007.

Nove Aldo, Puerto Plarta Market, Torino, Einaudi, 1997.

—, Superwoobinda e altre storie senza lieto fine, Torino, Einaudi, 1998.

Scurati Antonio, La letteratura dell'inesperienza. Scrivere romanzi al tempo della televisione, Milano, Bompiani, 2006.

Senardi Fulvio, Aldo Nove, Fiesole, Cadmo, 2005

Tondelli Pier Vittorio, Opere. Cronache, saggi, conversazioni, a cura di Fulvio Panzeri, Milano, Bompiani, 200I. 
